\title{
Geminivirus C2 protein represses genes involved in sulphur assimilation and this effect can be counteracted by jasmonate treatment
}

Rosa Lozano-Durán (1), Irene García (2), Stéphanie Huguet (3), Sandrine Balzergue (3), Luis C. Romero (2) and Eduardo R. Bejarano (1)

(1) Instituto de Hortofruticultura Subtropical y Mediterránea "La Mayora" (IHSMUMA-CSIC), Departamento de Genética, Facultad de Ciencias, Universidad de Málaga, 29071 Málaga, Spain

(2) Instituto de Bioquímica Vegetal y Fotosíntesis, Consejo Superior de Investigaciones Científicas and Universidad de Sevilla, 41092 Sevilla, Spain

(3) Unité de Recherche en Génomique Végétale (URGV), UMR INRA 1165 Université d'Evry Val d'Essonne - ERL CNRS 8196, 2 rue G. Crémieux, CP 5708, F91057 Evry Cedex, France

(4) Present address: The Sainsbury Laboratory, Norwich Research Park, Norwich, NR4 $7 \mathrm{UH}, \mathrm{UK}$

\begin{abstract}
Geminiviruses are plant viruses that infect a broad range of crops and cause extensive losses worldwide, having an important economic impact. $\mathrm{C} 2$, a multifunctional pathogenicity factor encoded by geminiviruses, has been recently shown to suppress the responses to jasmonates in the host plant, which might at least partially explain its wellestablished role in pathogenicity. Sulphur is one of the essential macro-elements for plant life, and is considered to have a role in plant defence, in a phenomenon named sulphur-induced resistance (SIR) or sulphur-enhanced defence (SED). In this work, we show that geminivirus $\mathrm{C} 2$ protein represses the expression of genes involved in the sulphur assimilation pathway in Arabidopsis, but, interestingly, this effect can be neutralized by exogenous jasmonate treatment. These preliminary results may raise the idea that geminiviruses might be affecting sulphur metabolism, and maybe counteracting SIR/SED, through the manipulation of the jasmonate signalling pathway, which would define a novel strategy in plant-virus interactions and may unveil SIR/SED as an important player in the plant defence against viruses.
\end{abstract}

Keywords Defence - Methyl-jasmonate - Sulphate - Sulphur-induced resistance (SIR) - Sulphur-enhanced defence (SED) - Virus - Sulphur metabolism - Pathogen

\section{Introduction}

Geminiviruses are plant viruses with circular, single-stranded DNA genomes (Rojas et al. 2005) that infect a wide range of plant species and cause extensive losses in crops 
worldwide, having an important economic impact. The members of this family have highly reduced genomes, encoding only six to eight proteins. Therefore, these viruses must rely on both their own limited multifunctional proteins and the host cell machinery to accomplish the infection, preventing or counteracting plant defence mechanisms at the same time (Hanley-Bowdoin et al. 2004).

C2 (also known as L2, AC2, AL2, or TrAP, for transcriptional activator protein) is a multifunctional pathogenicity factor encoded by geminiviruses. In viruses belonging to the genus Begomovirus, C2 acts as a transcription factor required for the expression of viral genes (Sunter and Bisaro 1992), and also triggers transactivation of host genes (Trinks et al. 2005). Additionally, C2 has been shown to suppress host defences: constitutive expression of truncated $\mathrm{C} 2$ from the begomovirus Tomato golden mosaic virus (TGMV) or the related L2 protein from the curtovirus Beet curly top virus (BCTV) in transgenic plants conditions an enhanced susceptibility phenotype (Sunter et al. 2001) that correlates with their ability to interact with and inactivate SNF1-related kinase (SnRK1) (Hao et al. 2003; Sunter et al. 2001). C2 and L2 are also gene silencing suppressors of both PTGS (post-transcriptional gene silencing) and TGS (transcriptional gene silencing) (reviewed in Raja et al. 2010).

Sulphur is one of the essential macro-elements for plant life, and it has numerous biological functions (Leustek et al. 2000). Plants are able to assimilate inorganic sulphur from the soil in form of sulphate; sulphate is reduced to sulphide and then incorporated into cysteine, a sulphur-containing organic molecule. Besides being an important proteinogenic amino acid, cysteine can be subsequently converted to a wide number of derived sulphur-containing compounds such as methionine, glucosinolates or phytoalexines. One of the most important cysteine-containing peptides is glutathione, a compound with multiple key roles, including protection against oxidative stress, heavy metals, or xenobiotics. Glutathione is an important regulator of redox signalling, and it has also been shown to be a player in plant defence operations (reviewed in Rausch and Wachter 2005). Other sulphur-containing compounds, such as phytoalexins or glucosinolates, are also considered to have a defensive role (and are generally referred to as SDCs or Sulphur-containing Defence Compounds) (reviewed in Rausch and Wachter 2005), and reactive sulphur species (RSS) play an important role in redox changes upon pathogen attack (reviewed in Gruhlke and Slusarenko 2012). As cysteine and glutathione content drops drastically upon limited sulphate availability (Nikiforova et al. 2003) and, on the contrary, pool sizes increase with excess sulphate, the importance of sulphur for plant defence becomes apparent. In agreement with this idea, it has been long known that high amounts of sulphur are able to suppress disease severity in fungus-infected plants (Bloem et al. 2004; Dubuis et al. 2005; Klikocka et al. 2005; Schnug et al. 1995), a phenomenon named sulphur-induced resistance (SIR) or sulphur-enhanced defence (SED) (reviewed in Rausch and Wachter 2005). Moreover, it has been recently demonstrated that Arabidopsis mutants impeded in cytosolic cysteine desulphhydrase activity show a systemic acquired resistance phenotype with high tolerance to biotrophic and necrotrophic pathogens (Alvarez et al. 2011) and that 
enhanced glutathione metabolism is correlated with SIR/SED to Tobacco mosaic virus (TMV) in tobacco plants (Holler et al. 2010).

Jasmonates are important plant signalling molecules that mediate both biotic and abiotic stress responses and regulate several aspects of growth and development. Plants respond to jasmonates by degrading the JAZ family of transcriptional repressors in a SCFCOI1complex- and a proteasome-dependent manner (Chini et al. 2007; Thines et al. 2007). Even though jasmonates have been traditionally linked to defence against necrotrophic pathogens, jasmonate treatment negatively impacts the infection by geminiviruses, and geminivirus $\mathrm{C} 2$ protein has been recently shown to suppress the responses to this plant hormone in the host plant (Lozano-Durán et al. 2011), which might at least partially explain its well-established role in pathogenicity.

In recent years, a link between sulphur metabolism and jasmonate signalling has been suggested by different authors. Nikiforova et al. 2003 and Hirai et al. 2003 showed that the jasmonate biosynthetic pathway is up-regulated in conditions of sulphur deficiency; Sasaki-Sekimoto et al. 2005 and Jost et al. 2005, on the other hand, proved that jasmonates induce the sulphur metabolic pathway. Recently, it has been reported that loss of synthesis of the metabolite S-sulfocysteine in the chloroplast results in high accumulation of reactive oxygen species (ROS) and repression of JA-regulated genes, although these effects are eliminated by reducing light conditions (Bermudez et al. 2010). These and other examples indicate that a mutual regulation exists between sulphur metabolism and jasmonate signalling, although the underlying mechanism still remains unknown.

In this work, we show that expression of geminivirus $\mathrm{C} 2$ protein represses genes involved in the sulphur assimilation pathway in Arabidopsis, but this effect can be neutralized by exogenous jasmonate treatment. These results suggest the possibility that geminiviruses might be counteracting SIR/SED through the manipulation of the jasmonate signalling pathway, which would define a novel strategy in plant-virus interactions.

\section{Materials and methods}

\section{Plant materials and growth conditions}

Transgenic Arabidopsis plants expressing Tomato yellow leaf curl Sardinia virus (TYLCSV) C2, Tomato yellow leaf curl virus (TYLCV) C2 or Beet curly top virus (BCTV) L2 have been described elsewhere (Lozano-Durán et al. 2011); expression and segregation analyses can be found in Lozano-Durán et al. 2011. The lines used in this work are C2-TS 9, C2-TM 1 and L2-BC 4. The T2 plants were selected in MS medium plus kanamycin and grown under short day conditions ( $8 \mathrm{~h}$ light/16 h dark).

For microarray analysis, T2 seedlings of transgenic Arabidopsis plants expressing C2 from TYLCSV (C2-TS 9) were grown on MS with kanamycin for 7 days, and then treated with $50 \mu \mathrm{M}$ MeJA or mock solution for $10 \mathrm{~h}$. Three biological independent 
replicates were performed. For these analyses, T3 homozygous LUC2 (PR1::LUC) transgenic plants (Murray et al. 2002) resistant to kanamycin were used as control. Previously, the hormonal response of LUC2 had been proven to be identical to that of the wild-type in the aforementioned assays (Lozano-Durán et al. 2011).

For gene expression studies and quantification of thiol compounds, fourteen-day-old seedlings were used.

\section{RT-PCR}

Primer pairs for RT-PCR were designed using Primer 3 software (http://frodo.wi.mit.edu/primer3/). Gene-specific primers were chosen so that the PCR products were 100-300 bp. Total RNA was extracted using RNAeasy Plant Mini Kit (Qiagen) and treated on column with Dnase (Qiagen). $1 \mu \mathrm{g}$ total RNA was used for first-strand cDNA synthesis using oligo(dT) primers and SuperScript II reverse transcriptase reagent (Invitrogen) following the manufacturer's instructions. The PCR conditions were: $5 \mathrm{~min}$ at $95{ }^{\circ} \mathrm{C}$, and 25 cycles of $30 \mathrm{~s}$ at $95^{\circ} \mathrm{C}, 30 \mathrm{~s}$ at $50{ }^{\circ} \mathrm{C}$ and $30 \mathrm{~s}$ at $72{ }^{\circ} \mathrm{C}$. The primers used were the following: for ATP sulphurylase, ATPS-F (CGAATGAAACAGCACGAGAA) and ATPS-R (GAGTCGTTCGAGTCCAGGAG); for APR1, APR1-F (GCAGGGGATTGAGAATTTGA) and APR1-R (TCTGGTCACCATCTGCTCTG); for APR3, APR3-F (CGAATGAAACAGCACGAGAA) and APR3-R (GAGTCGTTCGAGTCCAGGAG). Expression level of ATP-sulphurylase, APR1 and APR3 were normalized to actin. Actin (ACT2) was used as the internal control. Each cDNA sample used is a mixture from three biological replicates at a ratio 1:1:1.

\section{Real-time RT-PCR}

Quantitative real-time RT-PCR was used to analyze the expression of OAS-A1, OAS-B and OAS-C. First-strand cDNA was synthesized as described above. Gene-specific primers for each gene were designed using the Vector NTI Advance 10 software (Invitrogen; Table S1). Real-time PCR was performed using iQ SYBR Green Supermix (Bio-Rad), and the signals were detected on an iCYCLER (Bio-Rad) according to the manufacturer's instructions. The cycling profile consisted of $95{ }^{\circ} \mathrm{C}$ for $10 \mathrm{~min}$ followed by 45 cycles of $95{ }^{\circ} \mathrm{C}$ for $15 \mathrm{~s}$ and $60{ }^{\circ} \mathrm{C}$ for $1 \mathrm{~min}$. A melt curve from $60{ }^{\circ} \mathrm{C}$ to $90{ }^{\circ} \mathrm{C}$ was run following the PCR cycling. The expression levels of the genes of interest were normalized to that of the constitutive glycerol-3-phosphate dehydrogenase (GPDH) gene by subtracting the cycle threshold (CT) value of GPDH from the CT value of the gene $(\Delta \mathrm{CT})$. The fold change was calculated as $2-(\Delta \mathrm{CT}$ mutant - $\Delta \mathrm{CT}$ wild type). The sample of Col-0 wild type plants was used as the calibrator, with the expression level of the sample set to 1 (or $100 \%$ ). The results shown are means \pm SD of at least three RNA samples.

\section{Transcriptomic studies}


Seven-day-old LUC2 and transgenic C2-TS Arabidopsis seedlings were treated with a $50 \mu \mathrm{M}$ MeJA or mock solution for $10 \mathrm{~h}$. Total RNA was isolated from three biological replicates of MeJA- or mock-treated wild-type and transgenic C2-TS seedlings using TRIzol (Invitrogen) and subsequently cleaned using RNeasy MinElute Cleanup Kit (Invitrogen). All RNA samples were checked for their integrity on The Agilent 2100 bioanalyzer according to the Agilent Technologies (Waldbroon, Germany).

$1 \mu \mathrm{g}$ of total RNA was used to synthesize biotin-labelled cRNAs with the One-cycle cDNA synthesis kit (Affymetrix, Santa Clara, CA). Superscript II reverse transcriptase and T7-oligo (dT) primers were used to synthesize the single stranded cDNA at $42{ }^{\circ} \mathrm{C}$ during $1 \mathrm{~h}$ followed by the synthesis of the double stranded cDNA by using DNA ligase, DNA polymerase I and $\mathrm{RNaseH}$ during $2 \mathrm{~h}$ at $16{ }^{\circ} \mathrm{C}$. Clean up of the doublestranded cDNA was performed with Sample Cleanup Module (Affymetrix) followed by in vitro transcription (IVT) in presence of biotin-labeled UTP using GeneChip® IVT labelling Kit (Affymetrix). Quantity of the labelled-cRNA with RiboGreen ${ }^{\circledR}$ RNA Quantification Reagent (Turner Biosystems, Sunnyvale, CA) was determined after cleanup by the Sample Cleanup Module (Affymetrix). Fragmentation of $15 \mu \mathrm{g}$ of labelled-cRNA was carried out for $35 \mathrm{~min}$ at $94{ }^{\circ} \mathrm{C}$, followed by hybridization during 16 $\mathrm{h}$ at $45{ }^{\circ} \mathrm{C}$ to Affymetrix GeneChip ${ }^{\circledR}$ Arabidopsis genome array representing 22,500 probe sets corresponding to 24,000 gene sequences. After hybridization, the arrays were washed with two different buffers (stringent: 6X SSPE, $0.01 \%$ Tween-20 and nonstringent: $100 \mathrm{mM}$ MES, $0.1 \mathrm{M}[\mathrm{Na}+], 0.01 \%$ Tween-20) and stained with a complex solution including Streptavidin R-Phycoerythrin conjugate (Invitrogen/molecular probes, Carlsbad, CA) and anti Streptavidin biotinylated antibody (Vectors laboratories, Burlingame, CA). The washing and staining steps were performed in a GeneChip ${ }^{\circledR}$ Fluidics Station 450 (Affymetrix). The Affymetrix GeneChip® Arabidopsis Genome Arrays were finally scanned with the GeneChip ${ }^{\circledR}$ Scanner $30007 \mathrm{G}$ piloted by the GeneChip® Operating Software (GCOS).

All these steps were performed on Affymetrix platform at INRA-URGV from Evry. The raw CEL files were imported in $\mathrm{R}$ software for data analysis.

All raw and normalized data are available through the CATdb database (AFFY_MeJA_Arabidopsis, Gagnot et al. 2008) and from the Gene Expression Omnibus (GEO) repository at the National Center for Biotechnology Information (NCBI): accession number GSE18667.

For the data analysis, the data were normalized with the gcrma algorithm (Irizarry et al. 2003), available in the Bioconductor package (Gentleman and Carey 2002). To determine differentially expressed genes, we performed a usual two group t-test that assumes equal variance between groups. The variance of the gene expression per group is a homoscedastic variance, where genes displaying extremes of variance (too small or too large) were excluded. The raw $\mathrm{P}$ values were adjusted by the Bonferroni method, 
which controls the Family Wise Error Rate (FWER) (Ge et al. 2003). A gene is declared differentially expressed if the Bonferroni P-Value is less than 0.05 .

\section{Microarray analysis}

Analysis of the microarray data was performed using VirtualPlant 1.0 software (www.virtualplant.org) (Katari et al. 2010), and TAIR GO annotation tool (http://www.arabidopsis.org/tools/bulk/go/index.jsp).

\section{Quantification of thiol compounds}

To quantify the total cysteine and glutathione contents, thiols were extracted, reduced with $\mathrm{NaBH} 4$, and quantified by reverse-phase HPLC after derivatization with monobromobimane (Molecular Probes) as described previously (Dominguez-Solis et al. 2001).

\section{Statistical analysis}

For all the experiments shown, at least three independent samples were analyzed, unless otherwise indicated. ANOVA statistical analysis of data was performed using the program OriginPro 7.5 (OriginLab Corporation).

\section{Results}

Transcriptomic analysis of transgenic Arabidopsis plants expressing Tomato yellow leaf curl virus (TYLCSV) $\mathrm{C} 2$ reveals a repression of sulphur assimilation and jasmonate responses

Geminivirus C2 protein has been shown to interfere with the jasmonate signalling pathway (Lozano-Durán et al. 2011). With the aim of gaining insight into the effect of $\mathrm{C} 2$ on the response to jasmonates, we did a microarray analysis of Arabidopsis transgenic plants expressing C2 from TYLCSV (Lozano-Durán et al. 2011) in both basal conditions and after methyl-jasmonate (MeJA) treatment. Transgenic C2 or control seedlings were grown on plates with kanamycin for 7 days, and then treated with $50 \mu \mathrm{M}$ MeJA or mock solution for $10 \mathrm{~h}$; three biological and three technical replicates were used. After treatment, approximately 15 seedlings were harvested and total RNA was extracted. Total RNA from the three technical replicates in the three biological replicates was pooled and subsequently used for the microarray hybridizations. The up-regulation of well-characterized jasmonate-responsive genes upon MeJA treatment in the control plants proves the efficacy of the treatment (Table S1).

Intriguingly, this transcriptomic analysis unveiled the repression of three genes involved in sulphur assimilation in the untreated C2 plants: ATP-sulphurylase 3 (APS3; At4g14680; -2.48 fold), APS reductase 1 (APR1; At4g04610; -3.40 fold) and APS reductase 3 (APR3; At4g21990; -3.04 fold). The enzyme ATP-sulphurylase 3 catalyzes the activation of inorganic sulphate to form adenosine-5'-phosphosulphate (APS), which 
allows the reduction of the APS molecule to sulphite by the enzyme APS reductase, which uses glutathione as electron donor. Thermodynamically, both enzymes are biochemically linked and therefore regulated together (Yi et al. 2010). Although APS and APR are encoded by gene families in Arabidopsis, comprising four and three members respectively (APS1 to APS4 and APR1 to APR3), only APS3, APR1 and APR 3 seem to be repressed in $\mathrm{C} 2$ plants.

The microarray data and the position of the repressed genes in the assimilation metabolic pathway are depicted in Fig. 1A and B. The repression of APS3, APR1 and APR3 in the transgenic Arabidopsis plants was confirmed using semi-quantitative RTPCR (Fig. 1C).

In agreement with previous results (Lozano-Duran et al. 2011), the microarray data also revealed a repression of the jasmonate response in these transgenic plants. Consistently, functional enrichment analysis shows an over-representation of the Sulphur assimilation/Sulphur utilization and the Reponse to wounding GO categories among the whole subset of down-regulated genes in the $\mathrm{C} 2$ plants (Table 1).

In order to determine whether this $\mathrm{C} 2$-mediated repression of sulphur assimilation was a general mechanism exerted by geminiviruses, we evaluated the expression of APS3, APR1 and APR3 in transgenic Arabidopsis lines expressing C2 from two additional geminivirus species, Tomato yellow leaf curl virus (TYLCV) and BCTV (LozanoDurán et al. 2011). The RT-PCR results reveal a repression similar to that triggered by TYLCSV C2, suggesting that the repression of sulphur assimilation might be a general effect of geminivirus $\mathrm{C} 2$ protein (Fig. 1C).

\section{Transgenic Arabidopsis plants expressing geminivirus C2 accumulate reduced sulphur}

In general, high concentration of intracellular reduced sulphur (e. g. cysteine and glutathione) represses sulphur uptake and assimilation, and this repression takes place predominantly at the transcriptional level (reviewed in Buchner et al. 2004). For example, an Arabidopsis null mutant for the cysteine desulphhydrase enzyme shows a high content of total cysteine, and consequently several genes encoding sulphate transporters and APR are repressed (Alvarez et al. 2010). At the same time, cysteine and glutathione contents drop upon limited sulphate availability (Nikiforova et al. 2003). For this reason, we checked the levels of cysteine and glutathione in the transgenic Arabidopsis lines expressing geminivirus $\mathrm{C} 2$ in order to investigate whether differential accumulation of reduced sulphur might be affecting the expression of genes involved in sulphur assimilation. As shown in Fig. 2, cysteine and glutathione contents in transgenic seedlings expressing geminivirus $\mathrm{C} 2$ are higher than those in wild-type plants, and reach a similar value for the transgenic lines expressing three different geminivirus C2 proteins. To explore the possibility that this accumulation of reduced sulphur is the result of de novo synthesis, we evaluated the expression level of the cysteine biosynthetic O-acetylserine(thiol)lyase (OASTL) genes (OAS-A1, OAS-B and OAS-C) in our transgenic $\mathrm{C} 2$ plants using quantitative real-time PCR. Our results show that the 
expression of OAS-A1, OAS-B and OAS-C is not dramatically altered by $\mathrm{C} 2$ (all detected changes are <2-fold; Fig. 3), suggesting that the higher accumulation of cysteine is unlikely due to an increase in biosynthesis. Notably, the expression of OAS$\mathrm{C}$ is reduced in the transgenic $\mathrm{C} 2$ lines, and this reduction is statistically significant for plants expressing TYLCSV and TYLCV C2.

Jasmonate treatment restores the expression level of genes involved in sulphur assimilation in transgenic Arabidopsis plants expressing TYLCSV C2

Upon MeJA treatment, we observed dramatic transcriptional changes in wild-type Arabidopsis plants similar to those demonstrated in previous works (Jung et al. 2007; Nemhauser et al. 2006). Interestingly, we find that APR1, APR3 and APS3 are upregulated in response to the $10 \mathrm{~h}-\mathrm{MeJA}$ treatment in transgenic Arabidopsis plants expressing TYLCSV C2 when compared to mock-treated C2 plants (Table 2). No differences can be found in the expression level of these genes between MeJA-treated transgenic C2 plants and MeJA-treated control plants, indicating that this value is restored to the wild-type level following MeJA treatment.

\section{Discussion}

Genes involved in sulphur assimilation are repressed in compatible pathogen interactions and in transgenic plants expressing geminivirus $\mathrm{C2}$

Our results show that three genes involved in sulphur assimilation, APS3, APR1 and APR3, are repressed in transgenic Arabidopsis plants expressing geminivirus C2 protein. Since sulphur plays a role in plant defence, it would be interesting to check whether changes in expression of these genes are restricted to geminivirus-plant interactions or, on the contrary, are part of a general response to pathogens. Publicly available microarray data show that, notably, APS3, APR1 and APR3 are repressed in compatible Arabidopsis-pathogen interactions (with either the geminivirus Cabbage leaf curl virus - CaLCuV- (Ascencio-Ibanez et al. 2008), the RNA virus Turnip mosaic virus -TuMV- (Yang et al. 2007) or the pathogenic bacteria Pseudomonas syringae pv tomato -Pto-), whereas at least APR1 and APR3 are up-regulated in an incompatible interaction (with Pto expressing the avirulence factor AvrRpm1) (NASCARRAYS-120) (Table 3). These transcriptomic data, together with the correlation between an enhanced glutathione metabolism and SIR/SED to TMV in tobacco plants (Holler et al. 2010) suggest a possible role of sulphur metabolism, and maybe SIR/SED, in the interaction between plants and a wide array of pathogens, and not only fungi, as had been previously implied.

\section{Accumulation of reduced sulphur in transgenic C2 plants might be due to enhanced proteolysis}

Cysteine and glutathione quantifications in transgenic C2 plants show that both compounds accumulate to higher levels in the presence of geminivirus $\mathrm{C} 2$ protein, but the reason for this over-accumulation remains elusive. Bearing in mind that genes 
involved in sulphur uptake are repressed in transgenic C2 plants, it seems unlikely that the increase in reduced sulphur might come from enhanced sulphur assimilation, and it could rather derive from mobilization or re-assimilation of S-containing molecules and proteins. The expression of the OASTL genes in the $\mathrm{C} 2$ plants is in agreement with this idea. In a recent work, geminivirus $\mathrm{C} 2$ protein has been shown to inhibit the derubylating activity of the CSN complex at least over CULLIN1 (Lozano-Durán et al. 2011). In animals, down-regulation of the CSN has been described to enhance proteasomal proteolysis (Su et al. 2009). Therefore, it would be possible to speculate that the interference with the CSN activity caused by $\mathrm{C} 2$ could be promoting an enhancement of proteolysis in the plant cell, leading to higher levels of reduced sulhur available. In line with this idea, geminivirus infection induces the expression of components of the ubiquitin-proteasome system: 32 genes encoding proteasomal subunits are over-expressed in Arabidopsis in response to $\mathrm{CaLCuV}$ infection (AscencioIbanez et al. 2008), suggesting that proteolysis might be promoted in a geminivirusinfected plant.

\section{Could geminivirus $\mathrm{C} 2$ be counteracting sulphur-induced resistance through the suppression of jasmonate responses?}

Several data suggest that SIR/SED might play a role in plant-virus interactions: enhanced glutathione metabolism is correlated with SIR/SED to TMV in tobacco plants (Holler et al. 2010); genes involved in sulphur assimilation are repressed in infections with either DNA (CaLCuV) or RNA (TuMV) viruses (Ascencio-Ibanez et al. 2008; Yang et al. 2007); heterologous expression of the geminiviral pathogenicity determinant C2 also triggers a down-regulation of genes involved in sulphur assimilation (this work). Notably, we find a repression of genes involved in sulphur assimilation mediated by $\mathrm{C} 2$ from TYLCSV and TYLCV, two begomovirus from the Old World, and such a repression is detected during infection by $\mathrm{CaLCuV}$, a begomovirus from the New World (3). Moreover, we also detect a similar repression upon expression of L2, the homologous counterpart to $\mathrm{C} 2$, from the curtovirus BCTV. These results suggest the interesting idea that $\mathrm{C} 2$-mediated interference with the sulphur assimilation pathway at the transcriptional level might be a general strategy for geminiviruses. However, we should keep in mind that transgenic plants expressing pathogenicity factors constitutively and in all tissues, such as the ones used in this work, do not fully reflect the biology of the infection, and therefore these results, even if they could provide valuable hints for future studies, must be considered cautiously.

It is interesting to note that three out of four members of the APS family (APS1, APS3 and APS4) are targeted by microRNA395 (Kawashima et al. 2011), and their expression could in consequence be potentially affected by silencing suppressors, such as C2. Even though TYLCSV C2 is a rather weak silencing suppressor (Luna et al. 2012), has not been shown to bind miRNAs, and only seems to affect transcription of APS3, and not APS1 or APS4, we cannot rule out the possibility that C2 might be interfering with microRNA395 and in turn affecting the expression of these genes. 
Remarkably, the response to jasmonates is also repressed in the transgenic plants expressing TYLCSV C2 (Lozano-Durán et al. 2011; this work), and we showed that expression of genes involved in sulphur assimilation is restored to wild-type levels upon MeJA treatment. Interestingly, we found OAS-C, which is up-regulated by MeJA treatment (Arabidopsis eFP browser; Winter et al. 2007), to be repressed in the transgenic $\mathrm{C} 2$ lines. Even though we cannot rule out the possibility that the repression of genes involved in sulphur assimilation in the transgenic C2 Arabidopsis plants may be due to the accumulation of reduced sulphur, transcriptomic data support a direct link between jasmonate signalling and sulphur metabolism (our microarray data; Hirai et al. 2003; Jost et al. 2005; Nikiforova et al. 2003; Sasaki-Sekimoto et al. 2005), which leads us to postulate the existence of an additional mechanism, superimposed to the repression by product accumulation. Of note, the tantalizing idea that jasmonates may act as a linking signal between plant-pathogen interactions and sulphur metabolism has been suggested before (Kruse et al. 2007). Therefore, we hypothesize that geminivirus C2 protein might be affecting sulphur metabolism, and maybe counteracting SIR/SED, by triggering a suppression of the jasmonate response. However, the results obtained in this work are based on the use of transgenic lines, and should be confirmed in the context of a real infection; moreover, the higher accumulation of cysteine and glutathione in C2 plants does not clearly support a C2-mediated suppression of SIR/SED. Future experiments combining mutant viruses, controlled sulphur supply and hormone treatments will help unravel the potential effect of geminivirus $\mathrm{C} 2$ on sulphur metabolism and SID/SER, and might open a door towards new strategies in the fight against viral crop diseases.

\section{Acknowledgements}

The authors would like to thank Adela Zumaquero for her valuable help with the microarray data, and two anonymous reviewers for helpful suggestions. This research was supported by a grant from the Spanish Ministerio de Ciencia y Innovación/FEDER (AGL2007-66062-C02-02/AGR and AGL2010-22287-CO2) and by the European Regional Development Fund (ERDF) through the Ministerio de Ciencia e Innovación (grant no. BIO2010-15201) and the Junta de Andalucía (grant no. BIO-273). R.L-D. was awarded a Predoctoral Fellowship from the Spanish Ministerio de Educación y Cultura. 


\section{References}

Alvarez, C., Calo, L., Romero, L. C., Garcia, I., \& Gotor, C. (2010). An O-acetylserine(thiol)lyase homolog with L-cysteine desulfhydrase activity regulates cysteine homeostasis in Arabidopsis. Plant Physiology, 152, 656-669. doi:10.1104/pp.109.147975.

Alvarez, C., Angeles, B. M., Romero, L. C., Gotor, C., \& Garcia, I. (2011). Cysteine homeostasis plays an essential role in plant immunity. New Phytologist. doi:10.1111/j.1469-

8137.2011.03889.x.

Ascencio-Ibanez, J. T., Sozzani, R., Lee, T. J., Chu, T. M., Wolfinger, R. D., Cella, R., \& HanleyBowdoin, L. (2008). Global analysis of Arabidopsis gene expression uncovers a complex array of changes impacting pathogen response and cell cycle during geminivirus infection. Plant Physiology, 148, 436-454.

Bermudez, M. A., Paez-Ochoa, M. A., Gotor, C., \& Romero, L. C. (2010). Arabidopsis Ssulfocysteine synthase activity is essential for chloroplast function and long-day lightdependent redox control. The Plant Cell, 22, 403-416. doi:10.1105/tpc.109.071985.

Bloem, E., Riemenschneider, A., Volker, J., Papenbrock, J., Schmidt, A., Salac, I., Haneklaus, S., \& Schnug, E. (2004). Sulphur supply and infection with Pyrenopeziza brassicae influence Lcysteine desulphydrase activity in Brassica napus L. Journal of Experimental Botany, 55, 23052312. doi:10.1093/jxb/erh236erh236.

Buchner, P., Takahashi, H., \& Hawkesford, M. J. (2004). Plant sulphate transporters: coordination of uptake, intracellular and long-distance transport. Journal of Experimental Botany, 55, 1765-1773. doi:10.1093/jxb/erh206erh206.

Chini, A., Fonseca, S., Fernandez, G., Adie, B., Chico, J. M., Lorenzo, O., Garcia-Casado, G., Lopez-Vidriero, I., Lozano, F. M., Ponce, M. R., Micol, J. L., \& Solano, R. (2007). The JAZ family of repressors is the missing link in jasmonate signalling. Nature, 448, 666-671.

doi:10.1038/nature06006.

Dominguez-Solis, J. R., Gutierrez-Alcala, G., Vega, J. M., Romero, L. C., \& Gotor, C. (2001). The cytosolic O-acetylserine(thiol)lyase gene is regulated by heavy metals and can function in cadmium tolerance. Journal of Biological Chemistry, 276, 9297-9302.

Dubuis, P. H., Marazzi, C., Städler, E., \& Mauch, F. (2005). Sulphur deficiency causes a reduction in antimicrobial potential and leads to increased disease susceptibility of oilseed rape. Journal of Phytopathology, 153, 27-36.

Gagnot, S., Tamby, J. P., Martin-Magniette, M. L., Bitton, F., Taconnat, L., Balzergue, S., Aubourg, S., Renou, J. P., Lecharny, A., \& Brunaud, V. (2008). CATdb: a public access to Arabidopsis transcriptome data from the URGV-CATMA platform. Nucleic Acids Research, 36, 
D986-D990. doi:10.1093/nar/gkm757.

Ge, Y., Dudoit, S., \& Speed, T. P. (2003). Resampling-based multiple testing for microarray data analysis. TEST, 12, 1-44.

Gentleman, R., \& Carey, V. (2002). Bioconductor. RNews, 2, 1116.

Gruhlke, M. C., \& Slusarenko, A. J. (2012). The biology of reactive sulfur species (RSS). Plant Physiology and Biochemistry. doi:10.1016/j.plaphy.2012.03.016.

Hanley-Bowdoin, L., Settlage, S.B., Robertson, D. (2004). Reprogramming plant gene expression: a prerequisite to geminivirus DNA replication. Mol. Plant Pathol. 5.

Hao, L., Wang, H., Sunter, G., \& Bisaro, D. M. (2003). Geminivirus AL2 and L2 proteins interact with and inactivate SNF1 kinase. The Plant Cell, 15, 1034-1048.

Hirai, M. Y., Fujiwara, T., Awazuhara, M., Kimura, T., Noji, M., \& Saito, K. (2003). Global expression profiling of sulfur-starved Arabidopsis by DNA macroarray reveals the role of Oacetyl-I-serine as a general regulator of gene expression in response to sulfur nutrition. The Plant Journal, 33, 651-663.

Holler, K., Kiraly, L., Kunstler, A., Muller, M., Gullner, G., Fattinger, M., \& Zechmann, B. (2010). Enhanced glutathione metabolism is correlated with sulfur-induced resistance in Tobacco mosaic virus-infected genetically susceptible Nicotiana tabacum plants. Molecular PlantMicrobe Interactions, 23, 1448-1459. doi:10.1094/MPMI-05-10-0117.

Irizarry, R. A., Hobbs, B., Collin, F., Beazer-Barclay, Y. D., Antonellis, K. J., Scherf, U., \& Speed, T. P. (2003). Exploration, normalization, and summaries of high density oligonucleotide array probe level data. Biostatistics, 4, 249-264. doi:10.1093/biostatistics/4.2.2494/2/249.

Jost, R., Altschmied, L., Bloem, E., Bogs, J., Gershenzon, J., Hahnel, U., Hansch, R., Hartmann, T., Kopriva, S., Kruse, C., Mendel, R. R., Papenbrock, J., Reichelt, M., Rennenberg, H., Schnug, E., Schmidt, A., Textor, S., Tokuhisa, J., Wachter, A., Wirtz, M., Rausch, T., \& Hell, R. (2005). Expression profiling of metabolic genes in response to methyl jasmonate reveals regulation of genes of primary and secondary sulfur-related pathways in Arabidopsis thaliana. Photosynthesis Research, 86, 491-508. doi:10.1007/s11120-005-7386-8.

Jung, C., Lyou, S. H., Yeu, S., Kim, M. A., Rhee, S., Kim, M., Lee, J. S., Choi, Y. D., \& Cheong, J. J. (2007). Microarray-based screening of jasmonate-responsive genes in Arabidopsis thaliana. Plant Cell Reports, 26, 1053-1063. doi:10.1007/s00299-007-0311-1.

Katari, M. S., Nowicki, S. D., Aceituno, F. F., Nero, D., Kelfer, J., Thompson, L. P., Cabello, J. M., Davidson, R. S., Goldberg, A. P., Shasha, D. E., Coruzzi, G. M., \& Gutierrez, R. A. (2010). VirtualPlant: a software platform to support systems biology research. Plant Physiology, 152, 
500-515. doi:10.1104/pp.109.147025.

Kawashima, C. G., Matthewman, C. A., Huang, S., Lee, B. R., Yoshimoto, N., Koprivova, A., Rubio-Somoza, I., Todesco, M., Rathjen, T., Saito, K., Takahashi, H., Dalmay, T., \& Kopriva, S. (2011). Interplay of SLIM1 and miR395 in the regulation of sulfate assimilation in Arabidopsis. The Plant Journal, 66, 863-876. doi:10.1111/j.1365-313X.2011.04547.x.

Klikocka, H., Haneklaus, S., Bloem, E., \& Schnug, E. (2005). Influence of sulfur fertilization on infection of potato tubers with Rhizoctonia solani and Streptomyces scabies. J. Plant Nutr., 28, 819-833.

Kruse, C., Jost, R., Lipschis, M., Kopp, B., Hartmann, M., \& Hell, R. (2007). Sulfur-enhanced defence: effects of sulfur metabolism, nitrogen supply, and pathogen lifestyle. Plant Biology (Stuttgart, Germany), 9, 608-619. doi:10.1055/s-2007-965432.

Leustek, T., Martin, M. N., Bick, J. A., \& Davies, J. P. (2000). Pathways and Regulation of Sulfur Metabolism Revealed through Molecular and Genetic Studies. Annu Rev Plant Physiol Plant Mol Biol, 51, 141-165. doi:10.1146/annurev.arplant.51.1.141.

Lozano-Durán, R., Rosas-Díaz, T., Gusmaroli, G., Luna, A.P., Taconnat, L., Deng, X.W., Bejarano, E.R. (2011). Geminiviruses Subvert Ubiquitination by Altering CSN-Mediated Derubylation of SCF E3 Ligase Complexes and Inhibit Jasmonate Signaling in Arabidopsis thaliana. The Plant Cell 23.

Luna, A.P., Morilla, G., Voinnet, O., Bejarano, E.R. (2012) Functional analysis of gene silencing suppressors from Tomato yellow leaf curl disease viruses. Molecular Plant-Microbe Interactions, In press.

Murray, S. L., Thomson, C., Chini, A., Read, N. D., \& Loake, G. J. (2002). Characterization of a novel, defense-related Arabidopsis mutant, cir1, isolated by luciferase imaging. Molecular Plant-Microbe Interactions, 15, 557-566. doi:10.1094/MPMI.2002.15.6.557.

Nemhauser, J. L., Hong, F., \& Chory, J. (2006). Different plant hormones regulate similar processes through largely nonoverlapping transcriptional responses. Cell, 126, 467-475. doi:10.1016/j.cell.2006.05.050.

Nikiforova, V., Freitag, J., Kempa, S., Adamik, M., Hesse, H., \& Hoefgen, R. (2003). Transcriptome analysis of sulfur depletion in Arabidopsis thaliana: interlacing of biosynthetic pathways provides response specificity. The Plant Journal, 33, 633-650. 


\section{Figure captions}

Figure 1. a. Sulphur assimilation and sulphur-dependent generation of defence compounds (depicted in green). After uptake of sulphate from the external medium by high-affinity transporters in the roots (1), sulphate is transported and becomes activated through the activity of ATP sulphurylase in leaves (2). The product of this reaction, APS (5'-adenylsulphate) is reduced by an APS reductase (3); alternatively, APS can be further activated by an APS kinase (4) to form PAPS (3'-phosphoadenosin-5'phosphosulphate), required for various sulphatation reactions, including the biosynthesis of glucosinolates. Sulphite is reduced to sulphide by sulphite reductase (6), and incorporated into O-acetylserine to form cysteine. Cysteine is the primary product of S-assimilation, and is incorporated into sulphur-rich products (SRPs) and glutathione (GSH); furthermore, cysteine is also the donor of reduced sulphur for glucosinolate biosynthesis and for the synthesis of phytoalexins. Sulphide can be released from cysteine through the action of desulphhydrases, whereas elemental sulphur, S0, is probably released from GSH. Sulfate oxidase converts the excess sulphite to sulphate, releasing $\mathrm{H} 202$, which can act as a defence signal. (Modified from Rausch and Wachter 2005.) b. Genes involved in the sulphate assimilation down-regulated in the transgenic Arabidopsis plants expressing C2 from Tomato yellow leaf curl Sardinia virus according to the microarray data. The step of the sulphur assimilation pathway their products catalyze, the repression level and the corresponding p-value are indicated. c. Relative expression level of ATP-sulphurylase (APS3; At4g14680), APS reductase 1 (APR1; At4g04610), APS reductase 3 (APR3; At4g21990) or actin (AT3G18780), as a control, in transgenic C2 or wild-type Col-0 (WT) Arabidopsis 14-day-old seedlings by semi-quantitative RT-PCR. C2TS: Transgenic Arabidopsis plants expressing C2 from Tomato yellow leaf curl Sardinia virus. C2TM: Transgenic Arabidopsis plants expressing C2 from Tomato yellow leaf curl virus. L2BC: Transgenic Arabidopsis plants expressing C2 (L2) from Beet curly top virus. This experiment was repeated twice with similar results; results from one experiment are shown

Figure 2. Cysteine (Cys) and glutathione (GSH) content in $\mathrm{C} 2$ transgenic Arabidopsis seedlings. Fourteen-day-old seedlings expressing TYLCSV C2 (C2TS), TYLCV C2 (C2TYM) or BCTV L2 (L2BC), or wild-type (Col-0) Arabidopsis seedlings grown on MS medium were harvested, grinded and subjected to HPLC analysis. Total levels of cysteine and glutathione were measured. The values are the mean of five replicates, each corresponding to extracts obtained from at least five seedlings. Bars represent standard error. *, $\mathrm{P}<0.05$

Figure 3. OAS-A1, OAS-B and OAS-C steady-state transcription levels in transgenic Arabidopsis seedlings expressing TYLCSV C2 (C2TS), TYLCV C2 (C2TYM) or BCTV L2 (L2BC), measured by real-time quantitative real-time PCR, compared to the steady-state levels in wild-type Col-0 plants grown in parallel. The values are the mean of at least three replicates. Bars represent standard error. *, $\mathrm{P}<0.001$ 


\section{Table 1}

Table 1 Gene ontology analysis of down-regulated genes in transgenic Arabidopsis seedlings expressing C2 from Tomato yellow leaf curl Sardinia virus reveals an overrepresentation of the Sulphate assimilation/Sulphur utilization and the Response to wounding GO categories (a)

\begin{tabular}{|l|l|l|l|l|}
\hline GO term & $\begin{array}{l}\text { Observed } \\
\text { Frequency }\end{array}$ & $\begin{array}{l}\text { Expected } \\
\text { Frequency }\end{array}$ & p-value & Genes \\
\hline $\begin{array}{l}\text { Sulphate assimilation/Sulphur } \\
\text { utilization }\end{array}$ & $3.0 \%$ & $0.0 \%$ & 0.00075 & At4g21990 At4g14680 At4g04610 \\
\hline Response to wounding & $5.1 \%$ & $0.4 \%$ & 0.00261 & $\begin{array}{l}\text { At3g51660At2g34810At3g61190At4g23600 } \\
\text { At3g45140 }\end{array}$ \\
\hline
\end{tabular}

(a)The percentages of genes belonging to each category are reported for this subset of genes (observed frequency) and for the total number of genes present in the microarray (expected frequency). The p-value cutoff was 0.05 


\section{Table 2}

Table 2. Selected Gene Chip Hybridization data (a)

\begin{tabular}{|c|c|c|c|c|c|c|c|c|c|c|}
\hline AGI code & Gene & $\begin{array}{l}\text { C2 } \\
\text { MeJA }\end{array}$ & $\begin{array}{l}\text { C2 } \\
\text { mock }\end{array}$ & Ratio & $\begin{array}{l}\text { Ctrl } \\
\text { MeJA }\end{array}$ & $\begin{array}{c}\text { Ctrl } \\
\text { mock }\end{array}$ & Ratio & $\begin{array}{l}\text { C2 } \\
\text { MeJA }\end{array}$ & $\begin{array}{l}\text { Ctrl } \\
\text { MeJA }\end{array}$ & Ratio \\
\hline At4g14680 & APS3 & 9.53 & 6.78 & 2.75 & 9.47 & 8.09 & 1.38 & 9.53 & 9.47 & 0.06 \\
\hline At4g04610 & APR1 & 10.30 & 7.56 & 2.74 & 10.17 & 9.33 & 0.84 & 10.30 & 10.17 & 0.13 \\
\hline At4g21990 & APR3 & 10.30 & 8.77 & 1.53 & 10.72 & 10.38 & 0.34 & 10.30 & 10.72 & -0.42 \\
\hline
\end{tabular}

(a) Differential expression of ATP-sulphurylase (APS3; At4g14680), APS reductase 1 (APR1; At4g04610) and APS reductase 3 (APR3; At4g21990) in transgenic Arabidopsis seedlings expressing TYLCSV C2 (C2) or control (Crtl) seedlings after methyl jasmonate $(50 \mu \mathrm{M}$ MeJA, $10 \mathrm{~h})$ or mock treatment. Box data corresponding to $\log$ intensity hybridization are the result of the Log Intensities average of the three biological replicates. A positive ratio (grey boxes) indicates that the gene is up-regulated in plants expressing C2 compared to control plants after MeJA treatment. Ratios in black boxes were not found to be statistically significant after Bonferroni correction $(\mathrm{P}<0.05)$ 


\section{Table 3}

Table 3. Changes in the expression of ATP-sulphurylase (APS3; At4g14680), APS reductase 1 (APR1; At4g04610) and APS reductase 3 (APR3; At4g21990) in different plant-pathogen interactions, or in transgenic Arabidopsis seedlings expressing Tomato yellow leaf curl virus C2 (a)

\begin{tabular}{|c|c|c|c|c|c|}
\hline AGI & Gene & Microarray & $\log 2$ & $P$-value & Reference \\
\hline \multirow{5}{*}{ At4g04610 } & \multirow{5}{*}{ APS reductase $1(A P R I)$} & TYLCSV C2 & -1.77 & 0 & This work \\
\hline & & Pto & -2.43 & 0.014 & NASCARRAYS-120 \\
\hline & & AvrRpm1 & 3.18 & 0.001 & NASCARRAYS-120 \\
\hline & & $\mathrm{CaLCuV}$ & -0.5 & $1.69 \mathrm{E}-08$ & Ascencio-Ibanez et al. 2008 \\
\hline & & TuMV & Repressed & & Yang et al. 2007 \\
\hline \multirow{2}{*}{ At4g14680 } & \multirow{2}{*}{ ATP-sulphurylase (APS3) } & TYLCSV C2 & -1.31 & $1.27 \mathrm{E}-05$ & This work \\
\hline & & $\mathrm{CaLCuV}$ & -0.53 & $2.36 \mathrm{E}-12$ & Ascencio-Ibanez et al. 2008 \\
\hline \multirow{3}{*}{ At4g21990 } & \multirow{3}{*}{ APS reductase 3 (APR3) } & TYLCSV C2 & -1.60 & $7.27 \mathrm{E}-10$ & This work \\
\hline & & Pto & -2.25 & 0.024 & NASCARRAYS-120 \\
\hline & & AvrRpm1 & 3.47 & 0.0005 & NASCARRAYS-120 \\
\hline
\end{tabular}

(a) Pto: Infection with Pseudomonas syringae pv tomato. AvrRpm1: Infection with P. syringae pv tomato expressing the avirulence factor AvrRpm1. CaLCuV: Infection with the geminivirus Cabbage leaf curl virus. TuMV: Infection with the RNA virus Turnip mosaic virus. Infections with Pto, $\mathrm{CaLCuV}$ and TuMV are considered compatible interactions, whereas infection with AvrRpm1 is considered an incompatible interaction. NASCARRAYS-120: data are available at http://affy.arabidopsis.info/AffyList.html 


\section{Figure 1}

a

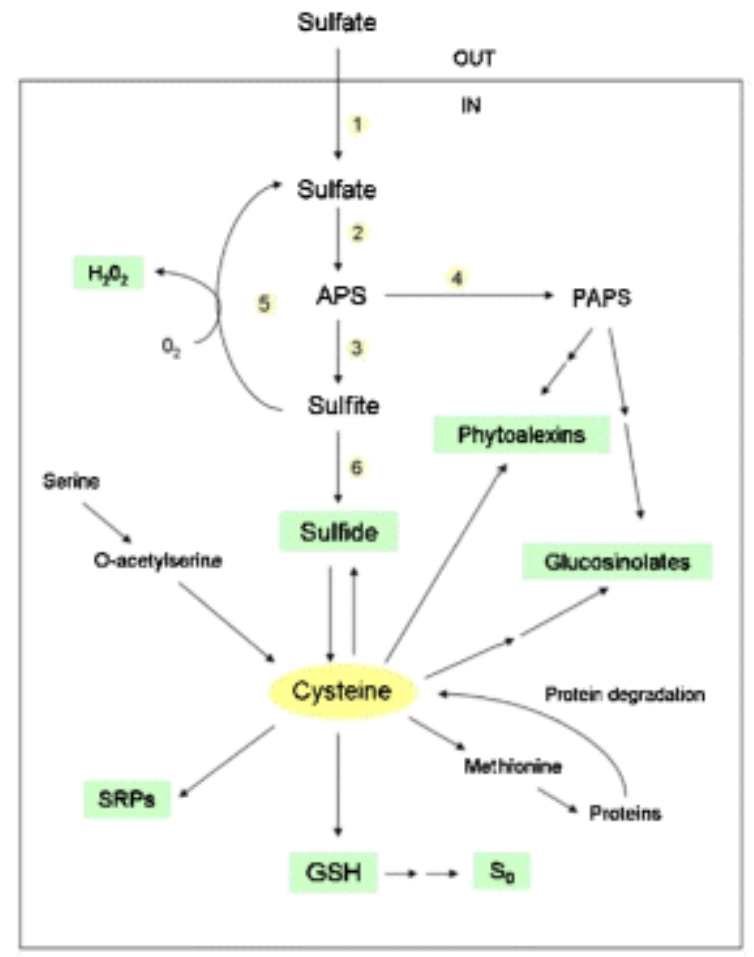

b

\begin{tabular}{ccccc}
\hline Step & AGI & Gene product & Log2 & P-value \\
\hline 2 & At4g14680 & ATP-sulfurylase (APS3) & $-1,31$ & $1,27 \mathrm{E}-05$ \\
3 & At4g04610 & APS reductase 1 (APR1) & $-1,77$ & $0,00 \mathrm{E}+00$ \\
3 & At4g21990 & APS reductase 3 (APR3) & $-1,6$ & $7,27 \mathrm{E}-10$ \\
\hline
\end{tabular}

C

WT C2TS C2TYM L2BC

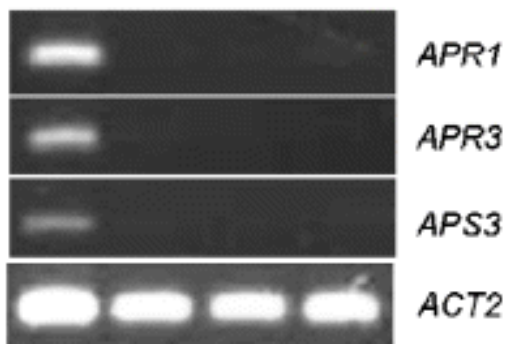


Figure 2
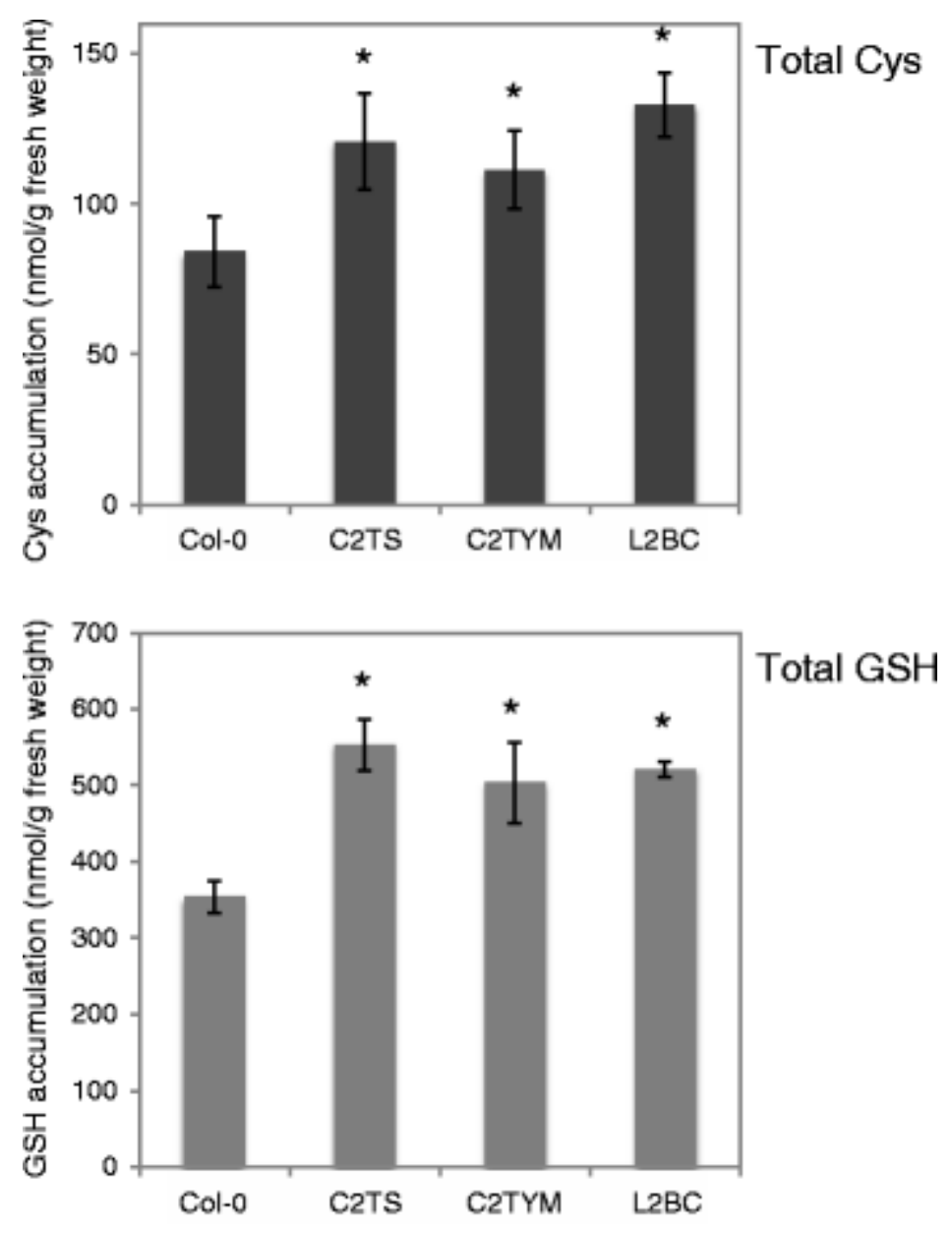
Figure 3

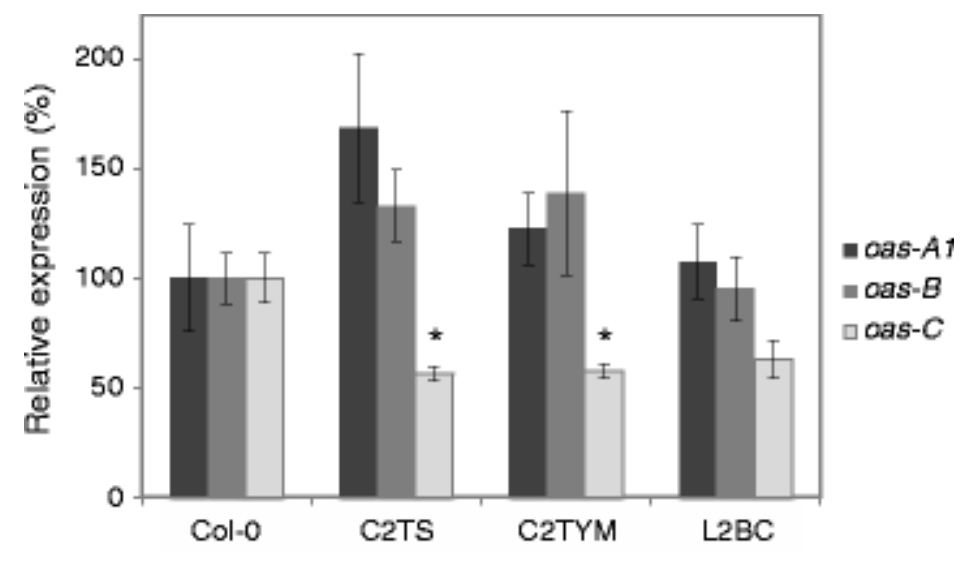

